\title{
Kobayashi non-hyperbolicity of Calabi-Yau manifolds via mirror symmetry
}

\author{
Ljudmila Kamenova1, Cumrun Vafa 2
}

\begin{abstract}
A compact complex manifold is Kobayashi non-hyperbolic if there exists an entire curve on it. Using mirror symmetry we establish that there are (possibly singular) elliptic or rational curves on any CalabiYau manifold $X$, whose mirror dual $\check{X}$ exists and is not "Hodge degenerate", therefore proving that $X$ is Kobayashi non-hyperbolic. We are not aware of any higher dimensional simply connected Calabi-Yau manifolds that satisfy the "Hodge degenerate" condition.
\end{abstract}

\section{Contents}

1 Introduction

2 Holomorphic Ray-Singer torsion and GW invariants 2

3 Non-hyperbolicity results 6

\section{Introduction}

In [K] S. Kobayashi conjectured that all compact Calabi-Yau manifolds have vanishing Kobayashi pseudometric. Kamenova-Lu-Verbitsky ([KLV]) proved Kobayashi's conjecture for all K3 surfaces and for certain compact hyperkähler manifolds that are deformation equivalent to Lagrangian fibrations. According to the hyperkähler SYZ conjecture, any compact hyperkähler manifold is deformation equivalent to a Lagrangian fibration.

A weaker version of Kobayashi's conjecture states that any compact Calabi-Yau manifold $X$ is Kobayashi non-hyperbolic, i.e., there is a nonconstant holomorphic map from $\mathbb{C}$ to $X$. This would be the case if there are

\footnotetext{
${ }^{1}$ Partially supported by a grant from the Simons Foundation/SFARI (522730, LK).

${ }^{2}$ The research of CV is supported in part by the NSF grant PHY-1067976 and by a grant from the Simons Foundation (602883, CV).
} 
(possibly singular) rational or elliptic curves on $X$. Using ergodicity of hyperkähler complex structures, in [V] M. Verbitsky showed that all compact hyperkähler manifolds are Kobayashi non-hyperbolic. Using mirror symmetry (more precisely, relating Ray-Singer torsion and genus one GromovWitten invarians) we show that a Calabi-Yau manifold $X$ is Kobayashi non-hyperbolic, assuming its mirror dual $\check{X}$ exists and satisfies some nondegeneracy condition involving Hodge bundles on the deformation space $\operatorname{Def}(\check{X})$ of $\check{X}$. Other than K3 surfaces and complex tori, we are not aware of other Calabi-Yau manifolds that satisfy the "Hodge degenerate" condition. Even though K3 surfaces and complex tori are "Hodge degenerate", they are Kobayashi non-hyperbolic. We suspect that, as Kobayashi conjectured, all Calabi-Yau manifolds are Kobayashi non-hyperbolic and that the "Hodge degenerate" condition is sufficient but not necessary.

In HBW D. R. Heath-Brown and P. Wilson have shown that for a Calabi-Yau threefold $X$ with a large Picard number $\rho(X)>13$ there is a birational morphism $\varphi: X \longrightarrow \bar{X}$ onto a normal projective threefold $\bar{X}$ with $\rho(\bar{X})<\rho(X)$ and such that the exceptional locus of $\varphi$ is covered by rational curves. This implies the existence of rational curves on $X$. In other words, $X$ is Kobayashi non-hyperbolic in the case of $\rho(X)>13$. However, in the cases with low Picard number there wouldn't be necessarily any smooth curves, but there could still be singular ones. Using mirror symmetry one can take into account singular elliptic or rational curves which is sufficient to imply non-hyperbolicity under the additional "Hodge non-degeneracy" condition.

\section{Holomorphic Ray-Singer torsion and GW in- variants}

In this paper we consider compact Calabi-Yau manifolds. A Calabi-Yau manifold $X$ is a compact complex Kähler manifold with a trival canonical bundle $\mathcal{K}_{X}$ and a trivial fundamental group $\pi_{1}(X)=0$. A hyperkähler manifold is a Calabi-Yau manifold with an everywhere non-degenerate holomorphic 2-form.

Let $X$ be a complex manifold. Recall that a pseudometric on $X$ is a function $d$ on $X \times X$ with values in $\mathbb{R}_{\geqslant 0}$ that satisfies all the properties of a distance function except for the non-degeneracy condition: $d(x, y)=0$ only if $x=y$. The Kobayashi pseudometric $d_{X}$ on $X$ is defined as the supremum 
of all pseudometrics $d$ on $X$ that satisfy the distance decreasing property with respect to holomorphic maps $f$ from the Poincaré disk $(\mathbb{D}, \rho)$ to $X$ :

$$
f^{*} d \leqslant \rho \text {, or equivalently, } d(f(x), f(y)) \leqslant \rho(x, y) \forall x, y \in \mathbb{D} .
$$

Here $\rho$ denotes the Poincaré metric on $\mathbb{D}$.

In [ $\mathrm{K}$ Kobayashi conjectured that if $X$ is a Calabi-Yau manifold, then $d_{X} \equiv 0$. Kamenova-Lu-Verbitsky ([KLV]) proved this conjecture for all K3 surfaces and for certain compact hyperkähler manifolds deformation equivalent to Lagrangian fibrations.

Definition 2.1: A compact manifold $X$ is called Kobayashi hyperbolic if any holomorphic map $\mathbb{C} \longrightarrow X$ is constant, or equivalently, the Kobayashi pseudometric is non-degenerate. A non-constant holomorphic map $\mathbb{C} \longrightarrow X$ is called an entire curve.

If the Kobayashi pseudometric is degenerate, the manifold $X$ is Kobayashi non-hyperbolic, i.e., there are entire curves on $X$. Examples of entire curves include (singular) rational or elliptic curves. Verbitsky has shown that all compact hyperkähler manifolds are Kobayashi non-hyperbolic, [V].

Definition 2.2: The holomorphic Ray-Singer torsion of a Calabi-Yau n-fold is

$$
T=\prod_{1 \leqslant p, q \leqslant n}\left(\operatorname{det} \Delta_{p, q}^{\prime}\right)^{(-1)^{p+q} p q}
$$

where $\Delta_{p, q}^{\prime}$ is the non-singular part of the $\bar{\partial}$-Laplace operator on the $(p, q)$ forms.

In the literature the holomorphic Ray-Singer torsion is also known as the BCOV torsion. Fang-Lu-Yoshikawa computed the BCOV invariant for quintic threefolds in $\mathbb{C} P^{4}$ explicitly (see Theorem 1.1 in [FLY]).

Let us recall the following formula from $[\mathrm{BCOV}]$ and $[\mathrm{FL}]$ :

Theorem 2.3: Let $\omega_{W P}$ and $\omega_{H^{i}}$ be the Kähler forms of the Weil-Peterson metric and the generalized Hodge metrics, respectively. Then

$$
\sum_{i=1}^{n}(-1)^{i} \omega_{H^{i}}-\frac{\sqrt{-1}}{2 \pi} \partial \bar{\partial} \log T=\frac{\chi(X)}{12} \omega_{W P}
$$


where $\chi(X)$ is the Euler characteristic of the Calabi-Yau manifold $X$.

Following Section 2 of $[\mathrm{FL}$ ] we define the terms in formula (2.2). For a polarized Calabi-Yau manifold $X$ there exists the universal family $\mathfrak{X}$ together with a proper surjective flat morphism $\pi: \mathfrak{X} \longrightarrow \operatorname{Def}(X)$. Let $\Omega$ be a nonzero holomorphic $(n, 0)$-form on $X$, where $n=\operatorname{dim}_{\mathbb{C}} X$. Consider the Hodge bundles $P R^{q} \pi_{*} \Omega_{\mathfrak{X} / \text { Def }}^{p} \rightarrow \operatorname{Def}(X)$, i.e., the relative version of the primitive cohomology groups $P H^{p, q}(X)$. Then for $1 \leqslant i \leqslant n$ we have (by Proposition 2.8 in $[\mathrm{FL}])$

$$
\omega_{H^{i}}=\sum_{1 \leqslant p \leqslant i} p c_{1}\left(R^{i-p} \pi_{*} \Omega_{\mathfrak{X} / \text { Def }}^{p}\right) .
$$

Similarly, the form of the Weil-Peterson metric is

$$
\omega_{W P}=c_{1}\left(R^{0} \pi_{*}\left(\Omega_{\mathfrak{X} / \text { Def }}^{n}\right)\right) .
$$

Remark 2.4: By the formulas above, the condition $\partial \bar{\partial} \log T=0$ can be expressed in terms of the first Chern classes of the Hodge bundles as

$$
\sum_{i=1}^{n} \sum_{p=1}^{i}(-1)^{i} p c_{1}\left(R^{i-p} \pi_{*} \Omega_{\mathfrak{X} / \text { Def }}^{p}\right)=\frac{\chi(X)}{12} c_{1}\left(R^{0} \pi_{*}\left(\Omega_{\mathfrak{X} / \text { Def }}^{n}\right)\right) .
$$

Definition 2.5: We call a Calabi-Yau manifold $X$ Hodge degenerate if it satisfies the condition (2.5) above, relating Chern classes of Hodge bundles.

Remark 2.6: In small dimensions there are simpler relations between the Kähler forms of the Hodge metric $\omega_{H}$ and the Weil-Peterson metric $\omega_{W P}$, namely if:

1. $n=2$, then $\omega_{H}=2 \omega_{W P}$;

2. $n=3$, then $\omega_{H}=(m+3) \omega_{W P}+\operatorname{Ric}\left(\omega_{W P}\right)$;

3. $n=4$, then $\omega_{H}=(2 m+4) \omega_{W P}+2 \operatorname{Ric}\left(\omega_{W P}\right)$,

where $n=\operatorname{dim} X$ and $m=\operatorname{dim} \operatorname{Def}(X)$ (see [BCOV] and [FL]).

Remark 2.7: Let $X$ be a Calabi-Yau 3-fold. Then the action of $T(\operatorname{Def}(X))$ on the lower degree Hodge bundles is trivial, hence $\omega_{H^{i}}=0$ for all $i<3$ (see [FL]). Therefore, the condition (2.5) becomes $-\omega_{H}=\frac{\chi(X)}{12} \omega_{W P}$. When 
we combine it with the second case of Remark 2.6, we obtain the following result.

Lemma 2.8: A Calabi-Yau 3-fold $X$ is Hodge degenerate if and only if $\operatorname{Ric}\left(\omega_{W P}\right)=-\left(m+3+\frac{\chi(X)}{12}\right) \omega_{W P}$, where $m=\operatorname{dim} \operatorname{Def}(X)=h^{1}\left(X, T_{X}\right)$.

Definition 2.9: Let $\overline{\mathfrak{M}}_{g, k}(X, d)$ denote the moduli space of stable degree $d$ maps from genus $g$ curves with $k$ marked points into a smooth projective variety $X$. Denote by $\left[\overline{\mathfrak{M}}_{g, k}(X, d)\right]^{\text {vir }}$ the virtual fundamental class of $\overline{\mathfrak{M}}_{g, k}(X, d)$.

Example 2.10: If $X$ is a quintic threefold, then the virtual fundamental class $\left[\overline{\mathfrak{M}}_{g, k}(X, d)\right]^{\text {vir }}$ is 0 -dimensional and its degree is

$$
N_{g, d}=\left\langle 1,\left[\overline{\mathfrak{M}}_{g, k}(X, d)\right]^{v i r}\right\rangle,
$$

which is called the degree $d$ genus $g$ Gromov-Witten invariant. In the genus 1 case, in $\overline{\mathfrak{M}}_{1, k}(X, d)$ one can define the main component $\overline{\mathfrak{M}}_{1, k}^{0}(X, d)$ as the closure of the locus in $\overline{\mathfrak{M}}_{1, k}(X, d)$ consisting of maps from smooth domains. The degree of the main component is the reduced genus 1 degree $d$ GWinvariant $N_{1, d}^{0}$. For a quintic threefold $X$, A. Zinger has established the following relation:

$$
N_{1, d}=N_{1, d}^{0}+\frac{1}{12} N_{0, d}
$$

(Theorem 1.1 in [Z1]). In particular, since $N_{1, d} \neq 0$, then at least one of $N_{1, d}^{0}$ or $N_{0, d}$ is non-zero, and therefore there will be genus one or genus zero curves on $X$, which implies that $X$ is Kobayashi non-hyperbolic.

Remark 2.11: Let $N_{1, d}$ be the degree $d$ genus one Gromov-Witten invariant of a quintic threefold $X$. A. Zinger has computed the generating function $\sum_{d=1}^{\infty} N_{1, d} e^{d t}$ on the A-side of $X$ (Theorem 1 in [Z2]). His computation coincides with Fang-Lu-Yoshikawa's computation of the BCOV torsion of the mirror dual $\check{X}$ of $X$ on the B-side, thus establishing that for quintic threefolds the number of elliptic curves on $X$ is related to the BCOV torsion of $\check{X}$ as predicted in Bershadsky-Cecotti-Ooguri-Vafa's paper [BCOV]. 


\section{Non-hyperbolicity results}

Here we establish Kobayashi non-hyperbolicity under the additional Hodge non-degeneracy assumption, using the tools of mirror symmetry. In this section we consider Calabi-Yau manifolds with maximal holonomy $S U(n)$. Recall that we defined $m:=\operatorname{dim} \operatorname{Def}(X)=h^{1}\left(X, T_{X}\right)$.

Theorem 3.1: Let $X$ be a Calabi-Yau 3-fold whose mirror dual $\check{X}$ exists and is not Hodge degenerate, i.e., $\operatorname{Ric}\left(\omega_{W P}\right) \neq-\left(m+3-\frac{\chi(X)}{12}\right) \omega_{W P}$. Then $X$ is Kobayashi non-hyperbolic.

Proof. Let $F_{1}$ be the topological string partition function of genus on 1 on $X$, i.e., the topological string amplitude. By [BCOV], $F_{1}$ corresponds to $\log T$, where $T$ is the holomorphic Ray-Singer torsion of the mirror dual $\check{X}$ of $X$. The contribution from constant maps in $F_{1}$ is $\frac{1}{24} \int k \wedge c_{2}$, where $k$ is the Kähler class. In order for $X$ to be Kobayashi non-hyperbolic (or more generally, to have non-constant holomorphic embeddings of genus 0 or 1 curves), we need that $F_{1}-\frac{1}{24} \int k \wedge c_{2} \neq 0$, for which it is enough that $\partial \bar{\partial} \log T \neq 0$ (see [BCOV]). If $\partial \bar{\partial} \log T \neq 0$, i.e., if $\check{X}$ is not Hodge degenerate, then there are rational or elliptic curves on $X$, i.e., $X$ is Kobayashi non-hyperbolic. Notice that since we are computing $\partial \bar{\partial} \log T$ for the mirror dual $\check{X}$ and $\chi(\check{X})=-\chi(X)$, then $\check{X}$ is not Hodge degenerate if and only if $\operatorname{Ric}\left(\omega_{W P}\right) \neq-\left(m+3-\frac{\chi(X)}{12}\right) \omega_{W P}$ by Lemma 2.8.

Similarly, if $X$ is a Calabi-Yau $n$-fold, the contribution from constant maps into $F_{1}$ is $\frac{(-1)^{n-1}}{24} \int k \wedge c_{n-1}$, where $k$ is the Kähler class, and for the condition

$$
F_{1}-\frac{(-1)^{n-1}}{24} \int k \wedge c_{n-1} \neq 0
$$

to be satisfied, it is enough to check that $\partial \bar{\partial} \log T \neq 0$, where $T$ is the holomorphic Ray-Singer torsion of the mirror dual $\check{X}$ of $X$. Therefore, in the general case we obtain the same result, however the condition that $\check{X}$ be Hodge non-degenerate in general is expressed in a more cumbersome way.

Theorem 3.2: Let $X$ be a Calabi-Yau $n$-fold $(n>2)$ whose mirror dual $\check{X}$ exists and is not Hodge degenerate, i.e., it does not satisfy equation (2.5). Then $X$ is Kobayashi non-hyperbolic.

\footnotetext{
${ }^{1}$ For example, in the case of a quintic threefold $F_{1}$ is the generating function with coefficients $N_{1, d}$, the degree $d$ genus one Gromov-Witten invariant of $X$.
} 
Remark 3.3: Notice that all K3 surfaces are Hodge degenerate, because for a K3 surface $S$ we have $\frac{\sqrt{-1}}{2 \pi} \partial \bar{\partial} \log T=\omega_{H}-\frac{\chi(S)}{12} \omega_{W P}=2 \omega_{W P}-\frac{24}{12} \omega_{W P}=0$ using the first case of Remark 2.6. However, K3 surfaces are Kobayashi nonhyperbolic as shown in [V], therefore the Hodge non-degenerate condition is sufficient, but not necessary. In higher dimensions $(n>2)$ we are not aware of any examples of simply connected Calabi-Yau manifolds with maximal holonomy that are Hodge degenerate. Complex tori, on the other hand, are Hodge degenerate, however they contain lots of entire curves, and therefore are Kobayashi non-hyperbolic.

Acknowledgements: The first named author would like to thank Aleksey Zinger and Zhiqin Lu for the interesting discussions and references. We are grateful to Albrecht Klemm and Dave Morrison for their comments and suggestions. We also thank Martin Roček and the SCGP for their hospitality during the Simons Summer Workshops.

\section{References}

[BCOV] Bershadsky, M., Cecotti, S., Ooguri, H., Vafa, C., Appendix by S. Katz, Holomorphic Anomalies in Topological Field Theories, Nucl. Phys. B 405 (1993) 279-304.

[CDFLL] Ceresole, A., D'Auria, R., Ferrara, S., Lerche, W., Louis, J., Picard-Fuchs Equations and Special Geometry, Int. J. Mod. Phys. A8 (1993) 79-114.

[FL] Fang, H., Lu, Z., Generalized Hodge metrics and BCOV torsion on Calabi-Yau moduli, J. reine angew. Math 588 (2005) 49-69.

[FLY] Fang, H., Lu, Z., Yoshikawa, K.-I., Analytic torsion for Calabi-Yau threefolds, J. Diff. Geom. 80 (2008) 175-259.

[HBW] Heath-Brown, D. R., Wilson, P., Calabi-Yau threefolds with $\rho>13$, Math. Ann. 294 (1992) 49-57.

[KLV] Kamenova, L., Lu, S., Verbitsky, M., Kobayashi pseudometric on hyperkahler manifolds, J. London Math. Soc. (2014) 90 (2): 436450. 
[K] Kobayashi, S., Intrinsic distances, measures and geometric function theory, Bull. Amer. Math. Soc. 82, no. 3 (1976) 357-416.

[V] Verbitsky, M., Ergodic complex structures on hyperkähler manifolds, Acta Math. 215 (2015) 161-182.

[Z1] Zinger, A., Reduced genus-one Gromov-Witten invariants, J. Diff. Geom. 83 (2009) 407-460.

[Z2] Zinger, A., The reduced genus 1 Gromov-Witten invariants of Calabi-Yau hypersurfaces, J. A. M. S. 22 (2009) 691-737.

LJUdmila Kamenova

Department of Mathematics, 3-115

STONy Brook University

Stony Brook, NY 11794-3651, USA,

kamenova@math.stonybrook.edu

Cumrun Vafa

JefFerson Physical Laboratory

HARVARD UNIVERSITY

Cambridge, MA 02138, USA,

vafa@physics.harvard.edu 